ISSN 1063-7737, Astronomy Letters, 2007, Vol. 33, No. 11, pp. 720-728. (C)Pleiades Publishing, Inc., 2007. Original Russian Text (c) V.V. Bobylev, A.T. Bajkova, 200\%, published in Pis'ma v Astronomicheskiu Zhurnal, 2007, Vol. 33, No. 11, pp. 809-818.

\title{
Galactic Rotation Parameters from Data on Open Star Clusters
}

\author{
V.V. Bobylev, A.T. Bajkova, and S.V. Lebedeva \\ Central (Pulkovo) Astronomical Observatory of RAS, St-Petersburg
}

\begin{abstract}
Currently available data on the field of velocities $V_{r}, V_{l}, V_{b}$ for open star clusters are used to perform a kinematic analysis of various samples that differ by heliocentric distance, age, and membership in individual structures (the Orion, Carina-Sagittarius, and Perseus arms). Based on 375 clusters located within $5 \mathrm{kpc}$ of the Sun with ages up to 1 Gyr, we have determined the Galactic rotation parameters $\omega_{0}=-26.0 \pm 0.3 \mathrm{~km} \mathrm{~s}^{-1} \mathrm{kpc}^{-1}$, $\omega_{0}^{\prime}=4.18 \pm 0.17 \mathrm{~km} \mathrm{~s}^{-1} \mathrm{kpc}^{-2}, \omega_{0}^{\prime \prime}=-0.45 \pm 0.06 \mathrm{~km} \mathrm{~s}^{-1} \mathrm{kpc}^{-3}$, the system contraction parameter $K=-2.4 \pm 0.1 \mathrm{~km} \mathrm{~s}^{-1} \mathrm{kpc}^{-1}$, and the parameters of the kinematic center $R_{0}=7.4 \pm 0.3 \mathrm{kpc}$ and $l_{0}=0 \pm 1^{\circ}$. The Galactocentric distance $R_{0}$ in the model used has been found to depend significantly on the sample age. Thus, for example, it is $9.5 \pm 0.7$ $\mathrm{kpc}$ and $5.6 \pm 0.3 \mathrm{kpc}$ for the samples of young $(\leq 50 \mathrm{Myr})$ and old $(>50 \mathrm{Myr})$ clusters, respectively. Our study of the kinematics of young open star clusters in various spiral arms has shown that the kinematic parameters are similar to the parameters obtained from the entire sample for the Carina-Sagittarius and Perseus arms and differ significantly from them for the Orion arm. The contraction effect is shown to be typical of star clusters with various ages. It is most pronounced for clusters with a mean age of $\approx 100 \mathrm{Myr}$, with the contraction velocity being $K r=-4.3 \pm 1.0 \mathrm{~km} \mathrm{~s}^{-1}$.
\end{abstract}

\section{INTRODUCTION}

The Galactic rotation parameters have been repeatedly determined by many authors using objects belonging to various Galactic structural components: from ionized and neutral hydrogen (Fich et al. 1989; Merrifield 1992; Brand and Blitz 1993; Nikiforov 1999; Avedisova 2005), from distant OB associations of stars (Dambis et al. 2001; Mel'nik et al. 2001), and from open star clusters (Zabolotskikh et al. 2002; Gerasimenko 2004; Popova and Loktin 2005b).

Open stars clusters (OSCs) are of great interest in studying the kinematics of the Galaxy, since they are located in a wide solar neighborhood and have reliable distance and age estimates.

Data only on the radial velocities $V_{r}$ (Mishurov et al. 1997; Gerasimenko 2004; Popova and Loktin 2005b) are commonly used for a kinematic analysis of OSCs, because the random errors in the radial velocities are essentially distance-independent and, hence, distant objects can be used. Note also that data only on the radial velocities are available for distant hydrogen clouds (Fich et al. 1989; Avedisova 2005). 
When such catalogs as Hipparcos (1997) and Tycho-2 (Hog et al. 2000) appeared, it became possible to accurately determine the mean proper motions of OSCs (Beshenov and Loktin 2004; Kharchenko et al. 2005b). A number of authors used simultaneously two observed velocity components, $V_{r}$ and $V_{l}$ (Mishurov and Zenina 1999; Dambis et al. 2001), or all three velocity components, $V_{r}, V_{l}$, and $V_{b}$ (Zabolotskikh et al. 2002; Bobylev 2004), to samples of stars within $3.4 \mathrm{kpc}$ of the Sun. In this paper, we also used all three observed velocity components.

One of the important problems is to determine the distance to the center of Galactic rotation $R_{0}$. This parameter is estimated indirectly from an analysis of the velocities of objects by reconciling the adopted model with observational data. Only in recent years has this parameter been estimated directly from objects of the Galactic nucleus (McNamara 2000; Eisenhauer et al. 2003). A summary of the $R_{0}$ values determined in the last decade by various methods can be found in Avedisova (2005) and an overview of the $R_{0}$ estimation methods is given in Nikiforov (2004).

The goal of this paper is to determine the Galactic rotation parameters $\left(\omega_{0}, \omega_{0}^{\prime}, \omega_{0}^{\prime \prime}\right)$ and the parameters of the kinematic center (Galactocentric distance $R_{0}$ and direction $l_{0}$ ) from currently available data on the field of space velocities $V_{r}, V_{l}$, and $V_{b}$, distances, and ages of OSCs and to study the kinematic peculiarities of various samples differing by heliocentric distance, age, and membership in individual arms.

\section{DATA}

At present, more than 1700 OSCs are known in the solar neighborhood. Data on their proper motions, radial velocities, and positions are needed for our purposes. A catalog that includes 652 OSCs (Kharchenko 2001; Kharchenko et al. 2005a, 2005b; Piskunov et al. 2006) forms the basis for our work list. The advantage of this catalog is a homogeneity and a high accuracy of the determination of mean cluster proper motions achieved by using the ASCC2.5 all sky catalog (Kharchenko 2001) compiled from Hipparcos (1997), Tycho-2 (Hog et al. 2000), and several other sources. The age estimates obtained by comparison with isochrones are available for the clusters of this catalog. The cluster distance estimates are based on the results by Loktin and Beshenov (2003), who reconciled the photometric estimates with the Hipparcos distance scale. We took other data from the compilation by Dias et al. (2002) and the WEBDA database (http://obsww.unige.ch/webda/).

For such open clusters as ASCC 16, ASCC 18, and $\operatorname{Tr} 10$, we used the mean radial velocities that we improved (Bobylev 2006) using the OSACA catalog (Bobylev et al. 2006a).

As a result, we compiled a database on the proper motions, radial velocities, and distances of 394 OSCs. They are located within about $5 \mathrm{kpc}$ of the Sun. Their ages do not exceed 1.5 Gyr. The radial velocities with estimates of their random errors are available for 270 $(\approx 70 \%$ of the sample) clusters. Note for comparison, that Dias and Lepine (2005) used only 212 OSCs with measured proper motions and radial velocities served as a source for their kinematic analysis.

To identify clusters belonging to various structures (e.g., the Orion, Perseus, and CarinaSagittarius arms), we use a probabilistic approach that we developed (Bobylev and Bajkova 2007). It is based on the approximation of the two-dimensional ( $X Y$ ) probability density function for the objects under consideration by a set of Gaussians that represent the proba- 
bility density functions of individual features.

\section{THE METHOD}

In this paper, we use a rectangular Galactic coordinate system with the axes directed away from the observer toward the Galactic center $\left(l=0^{\circ}, b=0^{\circ}\right.$, the $X$ axis), along the Galactic rotation $\left(l=90^{\circ}, b=0^{\circ}\right.$, the $Y$ axis $)$, and toward the North Galactic Pole $\left(b=90^{\circ}\right.$, the $Z$ axis).

The youngest disk objects that lie in the Galactic plane are commonly used to determine the Galactic rotation parameters. As a result, the observed velocity component $V_{b}$ is generally not considered, while the component of solar motion $w_{\odot}$ is assumed to be known (Dambis et al. 2001; Gerasimenko 2004). At the same time, analysis of relatively distant objects (Zabolotskikh et al. 2002; Bobylev 2004) showed that including the components $w_{\odot}$ and $V_{b}$ in the model allows their values to be also determined reliably. Therefore, we dwell on this more general approach. In the special case where the clusters are distributed in a ring (see the Carina-Sagittarius and Perseus sample below), we used a fixed value of $w_{\odot}=7.2 \mathrm{~km} \mathrm{~s}^{-1}$ (Dehnen and Binney 1998).

The method for determining the kinematic parameters used here consists in minimizing the quadratic functional $F$,

$$
\min F=\sum_{i=1}^{N} w_{r}^{i}\left(V_{r}^{i}-\hat{V}_{r}^{i}\right)^{2}+\sum_{i=1}^{N} w_{l}^{i}\left(V_{l}^{i}-\hat{V}_{l}^{i}\right)^{2}+\sum_{i=1}^{N} w_{b}^{i}\left(V_{b}^{i}-\hat{V}_{b}^{i}\right)^{2}
$$

under the following constraints derived from Bottlinger's formulas (Ogorodnikov 1965) with the angular velocity of Galactic rotation expanded in a series to terms of the second order of smallness in $r / R_{0}$ :

$$
\begin{gathered}
V_{r}=-u_{\odot} \cos b \cos \left(l-l_{0}\right)- \\
-v_{\odot} \cos b \sin \left(l-l_{0}\right)-w_{\odot} \sin b- \\
-R_{0}\left(R-R_{0}\right) \sin \left(l-l_{0}\right) \cos b \omega_{0}^{\prime}- \\
-0.5 R_{0}\left(R-R_{0}\right)^{2} \sin \left(l-l_{0}\right) \cos b \omega_{0}^{\prime \prime}+ \\
+r K \cos ^{2} b, \\
V_{l}=u_{\odot} \sin \left(l-l_{0}\right)-v_{\odot} \cos \left(l-l_{0}\right)- \\
-\left(R-R_{0}\right)\left(R_{0} \cos \left(l-l_{0}\right)-r \cos b\right) \omega_{0}^{\prime}- \\
-\left(R-R_{0}\right)^{2}\left(R_{0} \cos \left(l-l_{0}\right)-r \cos b\right) \times \\
\times 0.5 \omega_{0}^{\prime \prime}+r \omega_{0} \cos b \\
V_{b}=u_{\odot} \cos \left(l-l_{0}\right) \sin b+ \\
+v_{\odot} \sin \left(l-l_{0}\right) \sin b-w_{\odot} \cos b+ \\
+R_{0}\left(R-R_{0}\right) \sin \left(l-l_{0}\right) \sin b \omega_{0}^{\prime}+ \\
+0.5 R_{0}\left(R-R_{0}\right)^{2} \sin \left(l-l_{0}\right) \sin b \omega_{0}^{\prime \prime}- \\
-r K \cos b \sin b
\end{gathered}
$$

where $N$ is the number of clusters used; $i$ is the current cluster number; $V_{r, l, b}$ are the cluster velocities to be calculated, with $V_{r}$ being the radial velocity and $V_{l}=4.74 r \mu_{l} \cos b$ and $V_{b}=$ 
$4.74 \mu_{b}$ being the proper motion velocity components in the $l$ and $b$ directions, respectively (the coefficient 4.74 is the quotient of the number of kilometers in an astronomical unit by the number of seconds in a tropical year); $\hat{V}_{r}^{i}, \hat{V}_{l}^{i}, \hat{V}_{b}^{i}$ are the measured components of the velocity field; $w_{r}, w_{l}, w_{b}$ are the weights; $r$ is the heliocentric distance of the cluster calculated via the photometric parallax determined by bringing the cluster main sequence into coincidence with the corresponding isochrone (Piskunov et al. 2006); the cluster proper motion components $\mu_{l} \cos b$ and $\mu_{b}$ are in mas $\mathrm{yr}^{-1}$, the radial velocity $V_{r}$ is in $\mathrm{km} \mathrm{s}^{-1} ; u_{\odot}, v_{\odot}, w_{\odot}$ are the cluster centroid velocity components relative to the Sun; $R_{0}$ is the distance from the Sun to the kinematic center of the system; $R$ is the distance from the cluster to the center of rotation; $l_{0}$ is the direction of the kinematic center; $R, R_{0}$, and $r$ are in kpc. The quantity $\omega_{0}$ is the angular velocity of rotation at distance $R_{0}$, the parameters $\omega_{0}^{\prime}$ and $\omega_{0}^{\prime \prime}$ are the first and second-order derivatives of the angular velocity, respectively; $K$ is the Oort constant that describes the expansion/contraction of the stellar system. The distance $R$ can be calculated using the expression

$$
R^{2}=(r \cos b)^{2}-2 R_{0} r \cos b \cos \left(l-l_{0}\right)+R_{0}^{2} .
$$

Note also that Eqs. (2)-(4) are written in such a way that the direction of rotation from the $X$ axis to the $Y$ axis is positive.

The weights in functional (1) are assigned in accordance with the expressions (for simplification, the index $i$ was omitted)

$$
\begin{aligned}
& w_{r}=S_{0} / \sqrt{S_{0}^{2}+\sigma_{V_{r}}^{2}}, \\
& w_{l}=\beta^{2} S_{0} / \sqrt{S_{0}^{2}+\sigma_{V_{l}}^{2}}, \\
& w_{b}=\gamma^{2} S_{0} / \sqrt{S_{0}^{2}+\sigma_{V_{b}}^{2}},
\end{aligned}
$$

where $S_{0}$ denotes the dispersion averaged over all observations, which has the meaning of the "cosmic" dispersion that we take to be $8 \mathrm{~km} \mathrm{~s}^{-1} ; \beta=\sigma_{V_{r}} / \sigma_{V_{l}}$ and $\gamma=\sigma_{V_{r}} / \sigma_{V_{b}}$ are the scale factors (in our case, $\beta=1$ and $\gamma=2$ ). The errors in the velocities $V_{l}$ and $V_{b}$ can be calculated using the formula

$$
\sigma_{\left(V_{l}, V_{b}\right)}=\frac{4.74}{\pi} \sqrt{\mu_{l, b}^{2}\left(\frac{\sigma_{\pi}}{\pi}\right)^{2}+\sigma_{\mu_{l, b}}^{2}}
$$

where $\sigma_{\pi} / \pi$ is taken to be 0.2 , a typical error in the photometric parallax.

In this paper, apart from the system of weights (6) described above, we also use a variant of unit weights where $w_{r}=w_{l}=w_{b}=1$ for comparison.

The optimization problem (1)-(5) is solved for nine unknown parameters $u_{\odot}, v_{\odot}, w_{\odot}, \omega_{0}$, $\omega_{0}^{\prime}, \omega_{0}^{\prime \prime}, K, R_{0}$, and $l_{0}$ by a coordinate-wise descent method (the sought-for parameters are taken as the coordinates). According to this method, the increment $z_{x}$ for each unknown, which we arbitrarily denote by $x$, is sought from a necessary condition for the existence of an extremum of the functional $F\left(z_{x}\right)$ :

$$
\frac{d F\left(z_{x}\right)}{d z_{x}}=0
$$

The functional $F\left(z_{x}\right)$ is obtained by substituting Eqs. (2)-(4) for $V_{r}\left(z_{x}\right), V_{l}\left(z_{x}\right), V_{b}\left(z_{x}\right)$ into (1) using (5) in which the variable $x$ is replaced by $x+z_{x}$. 
Generally, Eq. (7) is a nonlinear equation for $z_{x}$. Its numerical solution by the Newton method is an iterative process. The value for $z_{x}$ at iteration $i$ is

$$
z_{x}^{i}=z_{x}^{i-1}-\left(d F\left(z_{x}\right) / d z_{x}\right) /\left.\left(d^{2} F\left(z_{x}\right) / d z_{x}^{2}\right)\right|_{z_{x}=z_{x}^{i-1}}
$$

The solution for $z_{x}$ usually converges after 1-3 iterations. As a result of one descent, the sought-for variable acquires a new value, $x+z_{x}$. In our case, about 1000 descents in all nine sought-for variables were required to obtain a solution with a sufficiently high accuracy.

A sufficient condition for the existence of a global extremum is that the Hessian matrix composed of elements $\left\{a_{i, j}\right\}=d^{2} F / d x_{i} d x_{j}$, where $x_{i}(i=1, \ldots 9)$ denote the sought-for parameters, be positively defined everywhere. We calculated the Hessian matrix in a wide domain of parameters: $4 \leq u_{\odot}, v_{\odot} \leq 16,2 \leq w_{\odot} \leq 11,-30 \leq \omega_{0} \leq 0,-3 \leq \omega_{0}^{\prime} \leq 6,-0.6 \leq$ $\omega_{0}^{\prime \prime} \leq 0.3,-4 \leq K \leq 5,3 \leq R_{0} \leq 15,-20 \leq l_{0} \leq 10$. Our analysis of the Hessian matrix for both weighting variants for all of the cluster samples considered here showed it to be positively defined. This suggests the existence of a global minimum in this domain and, as a result, the uniqueness of the solution. For unit weights, the Hessian matrix is also positively defined far outside this domain, which could not be established in the case of weighting according to rule (6). However, as will be shown below, the adopted weighting allowed the accuracy of the solutions obtained to be increased noticeably.

Interestingly, using weights of the form $w_{r, l, b}=1 / \sigma_{V_{r, l, b}}^{2}$ provided no positive definiteness of the Hessian matrix in the domain considered. It thus follows that several local extrema exist in this case. Therefore, to find the correct solution, we must know the initial approximation as accurately as possible. In contrast, if a global extremum exists, then we can start from any point of the domain. This, in particular, explains the modification of the weights according to rule (6), which provided a global minimum in a fairly wide domain of parameters.

We estimated the errors in the sought-for parameters by means of Monte-Carlo simulations. The errors were estimated by performing 100 cycles of computations. At this number of cycles, the mean values of the solutions virtually coincide with the solutions obtained only from the input data, i.e., without any addition of the measurement errors.

\section{CONSTRAINTS}

In solving the optimization problem (1)-(5), we used the following constraints on the data: (i) the magnitude of the peculiar velocity $\left|V_{p e c}\right|<90 \mathrm{~km} \mathrm{~s}^{-1}$; (ii) the cluster height above the Galactic plane $|z|<500 \mathrm{pc}$. The velocity $V_{\text {pec }}$ is calculated with respect to the local standard of rest $u_{\odot}=10.0 \mathrm{~km} \mathrm{~s}^{-1}, v_{\odot}=5.3 \mathrm{~km} \mathrm{~s}^{-1}, w_{\odot}=7.2 \mathrm{~km} \mathrm{~s}^{-1}$ (Dehnen and Binney 1998). According to constraint (i), four clusters were rejected. Constraint (ii) is important only for clusters older than 50 Myr. According to this criterion, the seven oldest clusters were rejected; all of the remaining clusters proved to be younger than 1 Gyr. After applying an additional constraint on the sample radius, $r<5 \mathrm{kpc}, 375$ clusters remained out the originally compiled list of 392 clusters, which served as the material for a further analysis. 


\section{RESULTS}

Figure 1 presents the spatial distribution of OSCs in the Galactic $X Y$ plane. Since a kinematic analysis of OSC samples with age separation is also the goal of this paper, we separately displayed the distributions of OSCs younger and older than 50 Myr in Figs. 1a and 1b, respectively. We see from Fig. 1a that young clusters clearly reproduce three segments of the spiral arms (Mel'nik et al. 2001; Dias and Lépine 2005; Popova and Loktin 2005a). The concentration regions are marked in the figure by the ellipses: the Perseus arm is on the left, the Orion arm is at the center, and the Carina-Sagittarius arm is on the right. We clearly see that the distribution of older clusters (Fig. 1b) is considerably more uniform, more compact, and concentrated closer to the Sun.

Figure 2 shows the mean errors $\sigma_{V_{r}}, \sigma_{V_{l}}$, and $\sigma_{V_{b}}$ as a function of the heliocentric distance. Note that in solving the optimization problem (1)-(5), we take into account the peculiarities of the error distribution using the weighting procedure (6).

Table 1 presents the results of our kinematic analysis of OSC samples with different sample radii $(r<5 \mathrm{kpc}$ and $r<2.5 \mathrm{kpc}$ ) and age separation (younger and older than 50 Myr) using the two weighting methods. Column 1 gives the sample type and size; column 2 lists the centroid velocity components relative to the Sun; columns 3, 4, and 5 list the rotation parameters; columns 6,7 , and 8 give the values of the $K$-effect, $R_{0}$, and $l_{0}$. The results obtained with unit weights and weights (6) are presented in the upper and lower parts of the table, respectively. As can be seen from Table 1, the random errors in virtually all parameters decrease when the system of weights (6) is used. For both weighting methods, the difference in $R_{0}$, which is significant for the sample of old clusters, is largest. As can be seen from Table 1, we obtain a discrepancy in $\omega_{0}^{\prime \prime}$ depending on the constraint on the sample radius. Figure 3 shows how the parameter $\omega_{0}^{\prime \prime}$ affects the Galactic rotation curve. To properly compare the results and to construct the curves indicated in Fig. 3, we obtained two solutions at fixed $R_{0}=7.4 \mathrm{kpc}$ :

$$
\begin{gathered}
\omega_{0}=-26.7 \pm 0.3 \mathrm{~km} \mathrm{~s}^{-1} \mathrm{kpc}^{-1} \\
\omega_{0}^{\prime}=4.27 \pm 0.11 \mathrm{~km} \mathrm{~s}^{-1} \mathrm{kpc}^{-2} \\
\omega_{0}^{\prime \prime}=-1.05 \pm 0.06 \mathrm{~km} \mathrm{~s}^{-1} \mathrm{kpc}^{-3},
\end{gathered}
$$

for the $r<2.5 \mathrm{kpc}$ sample and

$$
\begin{aligned}
& \omega_{0}=-26.0 \pm 0.2 \mathrm{~km} \mathrm{~s}^{-1} \mathrm{kpc}^{-1} \\
& \omega_{0}^{\prime}=4.14 \pm 0.10 \mathrm{~km} \mathrm{~s}^{-1} \mathrm{kpc}^{-2} \\
& \omega_{0}^{\prime \prime}=-0.44 \pm 0.04 \mathrm{~km} \mathrm{~s}^{-1} \mathrm{kpc}^{-3}
\end{aligned}
$$

for the $r<5 \mathrm{kpc}$ sample. As can be seen from Fig. 3, the difference in the rotation curve due to the influence of the second derivative becomes noticeable as one receded from $R_{0}$ to distances exceeding $\approx 1.5 \mathrm{kpc}$.

The Oort constants $A=0.5 R_{0} \omega_{0}^{\prime}$ and $B=\omega_{0}+0.5 R_{0} \omega_{0}^{\prime}$ calculated from the data in the fifth row of Table 1 are $A=15.4 \pm 0.6 \mathrm{~km} \mathrm{~s}^{-1} \mathrm{kpc}^{-1}$ and $B=-10.6 \pm 0.7 \mathrm{~km} \mathrm{~s}^{-1} \mathrm{kpc}^{-1}$ and have no significant differences for all solutions of Table 1.

In Fig. 4, linear contraction velocity $K r$ is plotted against heliocentric distance $r$ and mean sample age $t$. To obtain these characteristics, we divided the entire data set into four 
groups in such a way that each group contained approximately the same number of clusters. The dependences in Fig. 4 were derived at fixed Galactocentric distance $R_{0}=7.4 \mathrm{kpc}$. It follows from Fig. 4a that the contraction effect is traceable at all heliocentric distances $r>1$ $\mathrm{kpc}$ and is, on average, $K r=-3.2 \pm 1.0 \mathrm{~km} \mathrm{~s}^{-1}$. As we see from Fig. $4 \mathrm{~b}$, the contraction effect is typical of star clusters with various ages, but it is most pronounced for clusters with a mean age $t \approx 100 \mathrm{Myr}$; the contraction velocity in this case is $K r=-4.3 \pm 1.0 \mathrm{~km} \mathrm{~s}^{-1}$.

To study the kinematic peculiarities of various structures, we analyzed clusters younger than 50 Myr belonging to various arms (Fig. 1a). To identify the arms, we calculated the probability that each cluster belonged to one or another arm and then attributed it to the arm the probability of belonging to which was at a maximum. In this case, the probability densities of cluster belonging to each arm were fitted by Gaussians whose bases in the shape of ellipses are shown in Fig. 1b. The results of our kinematic analysis in which all parameters were found simultaneously are presented in the first four rows of Table 2 . The first and second two rows give, respectively, the results obtained with unit weights and with weights (6).

For the Carina-Sagittarius and Perseus sample, which contains mostly distant clusters, we also obtained a solution at fixed $w_{\odot}=7.2 \mathrm{~km} \mathrm{~s}^{-1}$ using weighting (6). This solution is given in the last row of Table 2 . It shows that a change in parameter $w_{\odot}$ by $\sim 2 \mathrm{~km} \mathrm{~s}^{-1}$ does not affect significantly the determination of other model parameters.

\section{DISCUSSION}

\section{Parameters of the Kinematic Center}

As can be seen from Table 1 , the values of $R_{0}$ obtained with various weighting methods agree well, within the $1 \sigma$ error limits. $R_{0}=7.4 \pm 0.3 \mathrm{kpc}$ that we found using clusters with a mixed (in age) composition is in good agreement with the $R_{0}$ estimates made by various authors.

Thus, for example, in the opinion of Avedisova (2005), the most probable value of $R_{0}$ lies within the range from 7.5 to $8.2 \mathrm{kpc}$. By analyzing the $R_{0}$ determinations by various authors, Nikiforov (2004) showed that the currently best value of $R_{0}$ is $7.9 \pm 0.2 \mathrm{kpc}$. Using 270 OSCs, Chen and Zhu (2007) obtained an estimate of $R_{0}=7.95 \pm 0.62 \mathrm{kpc}$.

Note that the Galactocentric distance $R_{0}=7.4 \pm 0.3 \mathrm{kpc}$ we found provides more evidence for the short distance scale (Dambis et al. 2001) than the IAU recommendations (1985), $R_{0}=8.5 \mathrm{kpc}$.

At the same time, as we see from Table. $1, R_{0}$ depends significantly on the sample age. Thus, for example, it is $R_{0}=9.5 \pm 0.7 \mathrm{kpc}$ for clusters younger than $50 \mathrm{Myr}$. On the other hand, a value of $R_{0}=7.1 \pm 0.6 \mathrm{kpc}$, which is close to that found from all 375 OSCs, was found from clusters younger than $50 \mathrm{Myr}$ that belong only to the Carina-Sagittarius and Perseus arms, but not to the Orion arm (Table 2). Our separate study of the Orion arm showed a significant difference almost in all kinematic parameters (see Table 2).

Clusters older than $50 \mathrm{Myr}$ are distributed fairly uniformly in space (Fig. 1b); the influence of the Galactic spiral structure and the Local system of stars in their motions is minor. One would think that they should be well suited to a reliable determination of $R_{0}$, but we found $R_{0}=5.6 \pm 0.3 \mathrm{kpc}$ for them (the lower part of Table 1). Based on a kinematic analysis of the OSC radial velocities from various catalogs and, in particular, the catalog by 
Piskunov et al. (2006), Nikiforov and Kazakevich (2006) point out a great uncertainty in $R_{0}$. In particular, they found $R_{0}=6 \pm 0.7 \mathrm{kpc}$ from a sample of old ( $\log t>8.8$ ) clusters, in good agreement with our result.

Analysis of the motions of the stars nearest the Sun showed that the vertex deviation for the youngest stars reaches $30^{\circ}$ (Dehnen and Binney 1998). Based on the clusters the results of whose analysis are presented in Table 1, we found no significant deviation from the direction $l_{0}=0^{\circ}$. This means that the rotation of the Galactic disk is nearly axisymmetric on large scales.

\section{The Galactic Rotation Curve}

The parameters of the Galactic rotation curve that we found in solution (8) at fixed $R_{0}=7.4$ $\mathrm{kpc}$ (which, in our case, is best suited to a proper comparison) are in good agreement with $\omega_{0}=-27.5 \pm 1.4 \mathrm{~km} \mathrm{~s}^{-1} \mathrm{kpc}^{-1}, \omega_{0}^{\prime}=4.54 \pm 0.24 \mathrm{~km} \mathrm{~s}^{-1} \mathrm{kpc}^{-2}, \omega_{0}^{\prime \prime}=-1.09 \pm 0.19 \mathrm{~km} \mathrm{~s}^{-1}$ $\mathrm{kpc}^{-3}$ determined from open clusters by Zabolotskikh et al. (2002) for the short distance scale and for $R_{0}=7.5 \mathrm{kpc}$. There is also good agreement with the results of our analysis of distant OB stars (Bobylev 2004): $\omega_{0}=-28.0 \pm 0.6 \mathrm{~km} \mathrm{~s}^{-1} \mathrm{kpc}^{-1}, \omega_{0}^{\prime}=4.17 \pm 0.14 \mathrm{~km} \mathrm{~s}^{-1}$ $\mathrm{kpc}^{-2}, \omega_{0}^{\prime \prime}=-0.81 \pm 0.12 \mathrm{~km} \mathrm{~s}^{-1} \mathrm{kpc}^{-3}$ (for $R_{0}=7.1 \mathrm{kpc}$ ).

We found the parameter $\omega_{0}^{\prime \prime}$ to decrease in absolute value with increasing sample radius. Far from the Sun, the Galactic rotation curve constructed from our data (Fig. 3) disagrees with the rotation curve constructed, for example, from the molecular gas (Avedisova 2005), which is flat in a wide $R$ range, from $2 \mathrm{kpc}$ to $15 \mathrm{kpc}$. Therefore, using a larger number of terms in the expansion of $\omega_{0}$ in terms of $r / R_{0}$ is of considerable interest in establishing the shape of the Galactic rotation curve from OSCs far from the Sun.

\section{The Contraction Velocity}

It is interesting to compare the contraction parameter $K=-2.4 \pm 0.1 \mathrm{~km} \mathrm{~s}^{-1} \mathrm{kpc}^{-1}$ that we found from all clusters with the results by Torra et al. (2000). Based on the OgorodnikovMilne linear model, these authors found $K=-2.0 \pm 0.4 \mathrm{~km} \mathrm{~s}^{-1} \mathrm{kpc}^{-1}$ for OB stars of all ages in the range of distances $100-2000 \mathrm{pc}$ and $K=-5.1 \pm 1.5 \mathrm{~km} \mathrm{~s}^{-1} \mathrm{kpc}^{-1}$ for OB stars with ages $t>60 \mathrm{Myr}$ in the range of distances 100-2000 pc. Fernández et al. (2001) and Bobylev et al. (2006b) showed that including the spiral structure did not eliminate the $K$ effect. Based on our results (Fig. 4), we can assume that the observed contraction velocity reflects the filling rate of the interarm space (Marochnik and Suchkov 1984), but a detailed study of this effect is outside the scope of our paper.

\section{Kinematic Peculiarities of the Arms}

In this paper, we applied no corrections for the influence of the spiral structure to the observed velocities. However, the influence of the spiral structure was studied in our previous paper (Bobylev et al. 2006b), where we showed that it has a major effect on the centroid velocity components relative to the Sun. This can explain the significant difference in the components $\Delta u_{\odot} \approx 2 \mathrm{~km} \mathrm{~s}^{-1}$ and $\Delta v_{\odot} \approx 5 \mathrm{~km} \mathrm{~s}^{-1}$ found from various arms.

Analysis of the kinematic parameters for young clusters belonging to the Orion arm indicates that such parameters as $\omega_{0}=-31.7 \pm 0.6 \mathrm{~km} \mathrm{~s}^{-1} \mathrm{kpc}^{-1}$ and $l_{0}=-3 \pm 1^{\circ}$ differ 
significantly from the Galactic rotation parameters found from all clusters. This shows that the Orion arm has an additional rotation with an angular velocity of $\approx 5 \mathrm{~km} \mathrm{~s}^{-1} \mathrm{kpc}^{-1}$ around a center that may not be associated with the Galactic center.

\section{CONCLUSIONS}

We compiled a database on 375 OSCs from various present-day catalogs and published sources. The clusters considered are located with $<5 \mathrm{kpc}$ of the Sun. Their ages do not exceed 1 Gyr.

The following Galactic rotation parameters were determined from data on the field of velocities $V_{r}, V_{l}, V_{b}$ for these OSCs: $\omega_{0}=-26.0 \pm 0.3 \mathrm{~km} \mathrm{~s}^{-1} \mathrm{kpc}^{-1}, \omega_{0}^{\prime}=4.18 \pm 0.17 \mathrm{~km} \mathrm{~s}^{-1}$ $\mathrm{kpc}^{-2}, \omega_{0}^{\prime \prime}=-0.45 \pm 0.06 \mathrm{~km} \mathrm{~s}^{-1} \mathrm{kpc}^{-3}$, the system contraction parameter $K=-2.4 \pm 0.1$ $\mathrm{km} \mathrm{s}^{-1} \mathrm{kpc}^{-1}$, and the parameters of the center of rotation $R_{0}=7.4 \pm 0.3 \mathrm{kpc}$ and $l_{0}=0^{\circ}$.

In addition, we performed a kinematic analysis of various OSC samples differing by heliocentric distance, age, and membership in individual arms. The value of $R_{0}$ was found to depend on the sample age. Thus, for example, it is $9.5 \pm 0.7 \mathrm{kpc}$ for clusters younger than $50 \mathrm{Myr}$ and $5.6 \pm 0.3 \mathrm{kpc}$ for clusters older than $50 \mathrm{Myr}$. Our study of the kinematics of young OSCs in various arms showed that the derived kinematic parameters are similar to the parameters obtained from the entire OSC sample for the Carina-Sagittarius arms and differ significantly from them for the Orion arm. The contraction effect was shown to be typical of star clusters with various ages and to be most pronounced for clusters with a mean age of $\approx 100 \mathrm{Myr}$; the linear contraction velocity in this case is $K r=-4.3 \pm 1.0 \mathrm{~km} \mathrm{~s}^{-1}$.

\section{ACKNOWLEDGMENTS}

We wish to thank I.I. Nikiforov for a discussion of our results and the referees for helpful remarks that contributed significantly to an improvement of the paper. This work was supported by the Russian Foundation for Basic Research (project no. 05-02-17047).

\section{REFERENCES}

1. V.S. Avedisova, Astron. Zh. 82, 488 (2005) [Astron. Rep. 49, 435 (2005)].

2. G.V. Beshenov and A.V. Loktin, Astron. Astrophys. Trans. 23, 103 (2004).

3. V.V. Bobylev, Pis'ma Astron. Zh. 30, 185 (2004) [Astron. Lett. 30, 159 (2004)].

4. V.V. Bobylev, Pis'ma Astron. Zh. 32, 906 (2006) [Astron. Lett. 32, 816 (2006)].

5. V.V. Bobylev, G.A. Goncharov, and A.T. Bajkova, Astron. Zh. 83, 821 (2006a) [Astron. Rep. 50, 733 (2006a)].

6. V.V. Bobylev, A.T. Bajkova, and G.A. Gontcharov, Astron. Astrophys. Trans. 25, 143 (2006b).

7. V.V. Bobylev and A.T. Bajkova, Pis'ma Astron. Zh. 33, 643 (2007) [Astron. Lett. 33, 571 (2007)].

8. J. Brand and L. Blitz, Astron. Astrophys. 275, 67 (1993).

9. M. Chen and Z. Zhu, Chin. J. Astron. Astrophys. 7, 120 (2007).

10. A.K. Dambis, A.M. Melnik, and A.S. Rastorguev, Pis'ma Astron. Zh. 27, 68 (2001) [Astron. Lett. 27, 58 (2001)]. 
11. W. Dehnen and J.J. Binney, Mon. Not. R. Astron. Soc. 298, 387 (1998).

12. W.S. Dias and J. R.D. Lépine, Astrophys. J. 629, 825 (2005).

13. W.S. Dias, B.S. Alessi, A. Moitinho, et al., Astron. Astrophys. 389, 971 (2002).

14. F. Eisenhauer, R. Schodel, R. Genzel, et al., Astrophys. J. 597, L121 (2003).

15. D. Fernández, F. Figueras, and J. Torra, Astron. Astrophys. 372, 833 (2001).

16. M. Fich, L. Blitz, and A.A. Stark, Astrophys. J. 342, 272 (1989).

17. T.P. Gerasimenko, Astron. Zh. 81, 124 (2004) [Astron. Rep. 48, 103 (2004)].

18. The HIPPARCOS and Tycho Catalogues, ES ASP- 1200 (1997).

19. E. Hog, C. Fabricius, V.V. Makarov, et al., Astron. Astrophys. 355, L27 (2000).

20. N.V. Kharchenko, Kinematika Fiz. Nebesnykh Tel 17, 409 (2001).

21. N.V. Kharchenko, A.E. Piskunov, S. Röser, et al., Astron. Astrophys. 438, 1163 (2005a).

22. N.V. Kharchenko, A.E. Piskunov, S. Röser, et al., Astron. Astrophys. 440, 403 (2005b).

23. A.V. Loktin and G.V. Beshenov, Astron. Zh. 80, 8 (2003) [Astron. Rep. 47, 6 (2003)].

24. L.S. Marochnik and A.A. Suchkov, Galaxies (Fizmatgiz, Moscow, 1984) [in Russian].

25. D.H. McNamara, J.B. Madsen, J. Barnes, et al., Publ. Astron. Soc. Pacif. 112, 202 (2000).

26. A.M. Mel'nik, A.K. Dambis, and A.S. Rastorguev, Pis'ma Astron. Zh. 27, 611 (2001) [Astron. Lett. 27, 521 (2001)].

27. M.R. Merrifield, Astron. J. 103, 1552 (1992).

28. Yu.N. Mishurov and I.A. Zenina, Astron. Astrophys. 341, 81 (1999).

29. Yu.N. Mishurov, I.A. Zenina, A.K. Dambis, et al., Astron. Astrophys. 323, 775 (1997).

30. I.I. Nikiforov, Astron. Zh. 76, 403 (1999) [Astron. Rep. 43, 345 (1999)].

31. I.I. Nikiforov, Astron. Soc. Pac. Conf. Ser. 316, 199 (2004).

32. I.I. Nikiforov and E.E. Kazakevich, Astron. Astrophys. Trans. 25, 189 (2006).

33. K.F. Ogorodnikov, Dynamics of Stellar Systems (Fizmatgiz, Moscow, 1958; Pergamon, Oxford, 1965).

34. A.E. Piskunov, N.V. Kharchenko, S. Röser, et al., Astron. Astrophys. 445, 545 (2006).

35. M.E. Popova and A.V. Loktin, Pis'ma Astron. Zh. 31, 190 (2005a) [Astron. Lett. 31, 171 (2005a)].

36. M.E. Popova and A.V. Loktin, Pis'ma Astron. Zh. 31, 743 (2005b) [Astron. Lett. 31, 663 (2005b)].

37. J. Torra, D. Fernández, and F. Figueras, Astron. Astrophys. 359, 82 (2000).

38. M.V. Zabolotskikh, A.S. Rastorguev, and A.K. Dambis, Pis'ma Astron. Zh. 28, 516 (2002) [Astron. Lett. 28, 454 (2002)].

Translated by V. Astakhov 
Table 1: Kinematic parameters of Galactic rotation

\begin{tabular}{|c|c|c|c|c|c|c|c|}
\hline Sample & $\begin{array}{c}u_{\odot}, v_{\odot}, w_{\odot}, \\
\mathrm{km} / \mathrm{s}\end{array}$ & $\begin{array}{c}\omega_{0}, \\
\mathrm{~km} / \mathrm{s} / \mathrm{kpc}\end{array}$ & $\begin{array}{c}\omega_{0}^{\prime} \\
\mathrm{km} / \mathrm{s} / \mathrm{kpc}^{2}\end{array}$ & $\begin{array}{c}\omega_{0}^{\prime \prime} \\
\mathrm{km} / \mathrm{s} / \mathrm{kpc}^{3}\end{array}$ & $\begin{array}{c}K \\
\mathrm{~km} / \mathrm{s} / \mathrm{kpc}\end{array}$ & $\begin{array}{l}R_{0}, \\
\mathrm{kpc}\end{array}$ & $\begin{array}{l}l_{0} \\
\text { deg. }\end{array}$ \\
\hline $\begin{array}{c}r<5 \\
\mathrm{kpc} \\
(\mathrm{N}=375)\end{array}$ & $\begin{array}{r}9.5_{(0.4)} \\
10.9_{(0.3)} \\
9.1_{(0.2)} \\
\end{array}$ & $-26.7_{(0.4)}$ & $3.91_{(0.21)}$ & $-0.35_{(0.08)}$ & $-2.3_{(0.1)}$ & $8.0_{(0.4)}$ & $0_{(1)}$ \\
\hline $\begin{array}{c}r<2.5 \\
\mathrm{kpc} \\
(\mathrm{N}=340)\end{array}$ & $\begin{array}{r}9.2_{(0.3)} \\
11.9_{(0.3)} \\
8.4_{(0.2)}\end{array}$ & $-27.2_{(0.5)}$ & $3.72_{(0.32)}$ & $-0.70_{(0.13)}$ & $-2.6_{(0.2)}$ & $8.5_{(0.7)}$ & $-2_{(1)}$ \\
\hline $\begin{array}{c}t \leq 50 \\
\mathrm{Myr} \\
(\mathrm{N}=196)\end{array}$ & $\begin{array}{r}9.6_{(0.4)} \\
11.7_{(0.4)} \\
9.4_{(0.3)} \\
\end{array}$ & $-27.4_{(0.5)}$ & $3.21_{(0.25)}$ & $-0.14_{(0.07)}$ & $-1.4_{(0.2)}$ & $9.8_{(0.7)}$ & $0_{(1)}$ \\
\hline $\begin{array}{c}t>50 \\
\mathrm{Myr} \\
(\mathrm{N}=178)\end{array}$ & $\begin{array}{r}9.7_{(0.4)} \\
10.2_{(0.4)} \\
8.8_{(0.4)} \\
\end{array}$ & $-25.3_{(0.9)}$ & $4.59_{(0.60)}$ & $-0.59_{(0.19)}$ & $-3.5_{(0.3)}$ & $6.7_{(0.8)}$ & $0_{(1)}$ \\
\hline $\begin{array}{c}r<5 \\
\mathrm{kpc} \\
(\mathrm{N}=375)\end{array}$ & $\begin{array}{r}9.2_{(0.2)} \\
10.7_{(0.2)} \\
8.2_{(0.2)}\end{array}$ & $-26.0_{(0.3)}$ & $4.18_{(0.17)}$ & $-0.45_{(0.06)}$ & $-2.4_{(0.1)}$ & $7.4_{(0.3)}$ & $0_{(1)}$ \\
\hline $\begin{array}{c}r<2.5 \\
\mathrm{kpc} \\
(\mathrm{N}=340)\end{array}$ & $\begin{array}{r}8.8_{(0.2)} \\
12.0_{(0.2)} \\
8.0_{(0.1)}\end{array}$ & $-26.7_{(0.3)}$ & $3.45_{(0.26)}$ & $-0.69_{(0.11)}$ & $-2.4_{(0.1)}$ & $9.0_{(0.7)}$ & $-2_{(1)}$ \\
\hline $\begin{array}{c}t \leq 50 \\
\mathrm{Myr} \\
(\mathrm{N}=196)\end{array}$ & $\begin{array}{r}9.2_{(0.3)} \\
11.5_{(0.3)} \\
8.6_{(0.2)} \\
\end{array}$ & $-26.7_{(0.4)}$ & $3.24_{(0.22)}$ & $-0.19_{(0.07)}$ & $-1.4_{(0.2)}$ & $9.5_{(0.7)}$ & $0_{(1)}$ \\
\hline $\begin{array}{c}t>50 \\
\mathrm{Myr} \\
(\mathrm{N}=178)\end{array}$ & $\begin{array}{r}9.4_{(0.3)} \\
10.0_{(0.2)} \\
7.8_{(0.2)} \\
\end{array}$ & $-25.5_{(0.5)}$ & $5.57_{(0.37)}$ & $-0.89_{(0.15)}$ & $-3.5_{(0.2)}$ & $5.6_{(0.3)}$ & $-1_{(1)}$ \\
\hline
\end{tabular}


Table 2: Kinematic parameters of young ( $<50$ Myr) OSCs in the arms

\begin{tabular}{|c|c|c|c|c|c|c|c|}
\hline Arm & $\begin{array}{c}u_{\odot}, v_{\odot}, w_{\odot} \\
\mathrm{km} / \mathrm{s}\end{array}$ & $\begin{array}{c}\omega_{0}, \\
\mathrm{~km} / \mathrm{s} / \mathrm{kpc}\end{array}$ & $\begin{array}{c}\omega_{0}^{\prime}, \\
\mathrm{km} / \mathrm{s} / \mathrm{kpc}^{2}\end{array}$ & $\begin{array}{c}\omega_{0}^{\prime \prime}, \\
\mathrm{km} / \mathrm{s} / \mathrm{kpc}^{3}\end{array}$ & $\begin{array}{c}K \\
\mathrm{~km} / \mathrm{s} / \mathrm{kpc} \\
\end{array}$ & $\begin{array}{l}R_{0} \\
\mathrm{kpc}\end{array}$ & $\begin{array}{r}l_{0} \\
\text { deg. }\end{array}$ \\
\hline $\begin{array}{c}\text { Car-Sag } \\
\text { and Perseus } \\
\quad(\mathrm{N}=92)\end{array}$ & $\begin{array}{l}9.0_{(0.7)} \\
9.6_{(1.4)} \\
9.5_{(0.4)} \\
\end{array}$ & $-27.3_{(0.6)}$ & $4.04_{(0.41)}$ & $-0.37_{(0.16)}$ & $-1.8_{(0.4)}$ & $8.3_{(1.0)}$ & $1_{(1)}$ \\
\hline $\begin{array}{c}\text { Orion } \\
(\mathrm{N}=93)\end{array}$ & $\begin{array}{r}8.7_{(1.0)} \\
14.2_{(0.4)} \\
9.5_{(0.3)} \\
\end{array}$ & $-31.6_{(1.0)}$ & $4.46_{(0.84)}$ & $0.23_{(0.38)}$ & $-0.5_{(0.6)}$ & $5.4_{(0.9)}$ & $-3_{(2)}$ \\
\hline $\begin{array}{c}\text { Car-Sag } \\
\text { and Perseus } \\
(\mathrm{N}=92)\end{array}$ & $\begin{array}{l}6.5_{(0.5)} \\
8.7_{(1.1)} \\
9.1_{(0.4)} \\
\end{array}$ & $-25.8_{(0.5)}$ & $4.52_{(0.37)}$ & $-0.70_{(0.17)}$ & $-2.6_{(0.4)}$ & $7.1_{(0.6)}$ & $1_{(1)}$ \\
\hline $\begin{array}{c}\text { Orion } \\
(\mathrm{N}=93)\end{array}$ & $\begin{array}{r}8.7_{(0.5)} \\
14.0_{(0.4)} \\
8.3_{(0.3)} \\
\end{array}$ & $-31.7_{(0.6)}$ & $5.14_{(0.65)}$ & $-0.20_{(0.34)}$ & $-0.5_{(0.4)}$ & $4.9_{(0.5)}$ & $-3_{(1)}$ \\
\hline $\begin{array}{c}\text { Car-Sag } \\
\text { and Perseus } \\
\qquad(\mathrm{N}=92)\end{array}$ & $\begin{array}{l}6.5_{(0.6)} \\
8.7_{(1.0)} \\
-\end{array}$ & $-25.9_{(0.5)}$ & $4.55_{(0.38)}$ & $-0.69_{(0.17)}$ & $-2.6_{(0.4)}$ & $7.1_{(0.7)}$ & $1_{(1)}$ \\
\hline
\end{tabular}

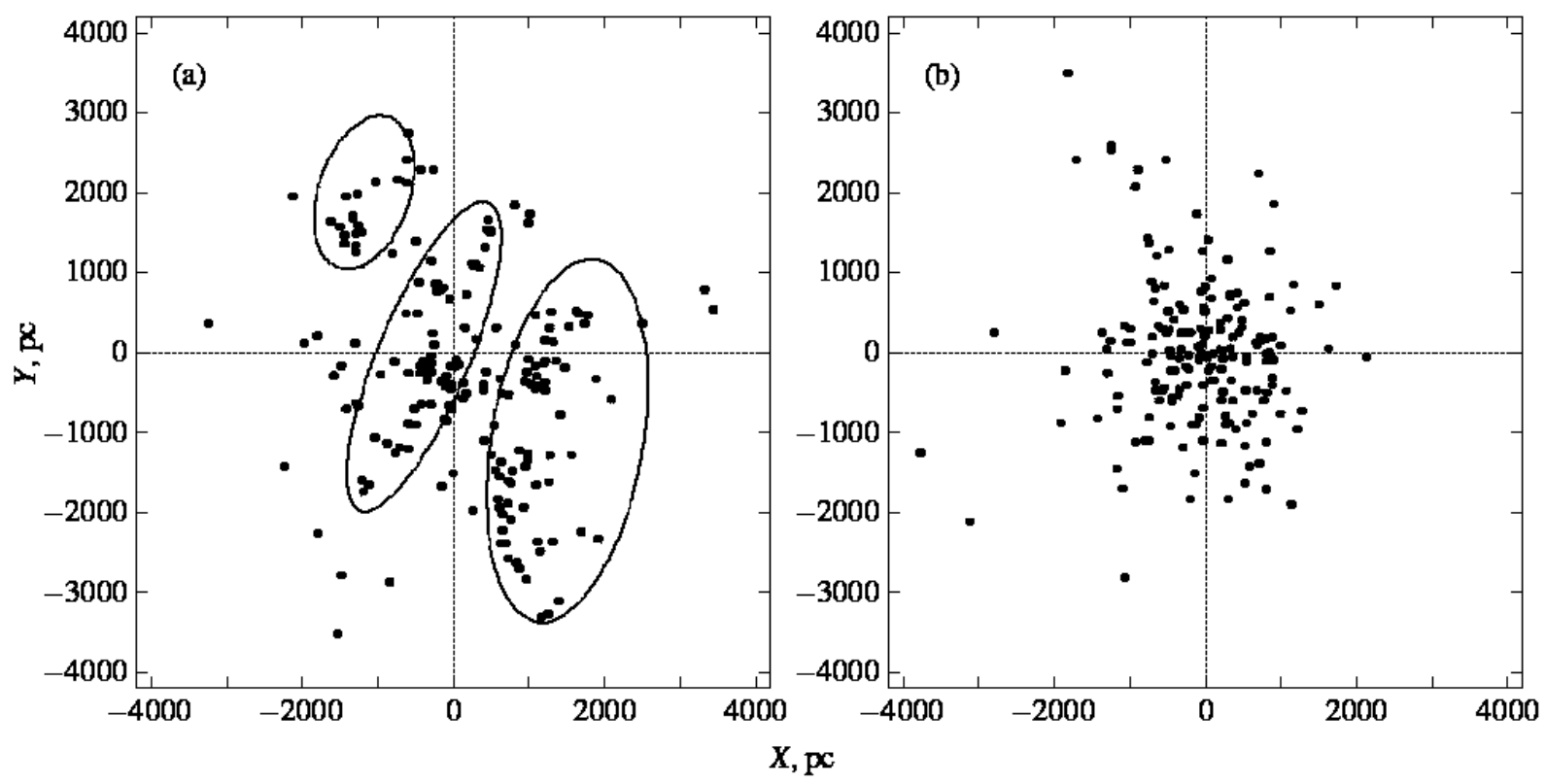

Fig. 1. Distribution of OSCs (a) younger than 50 Myr and (b) older than 50 Myr in the Galactic $X Y$ plane. 


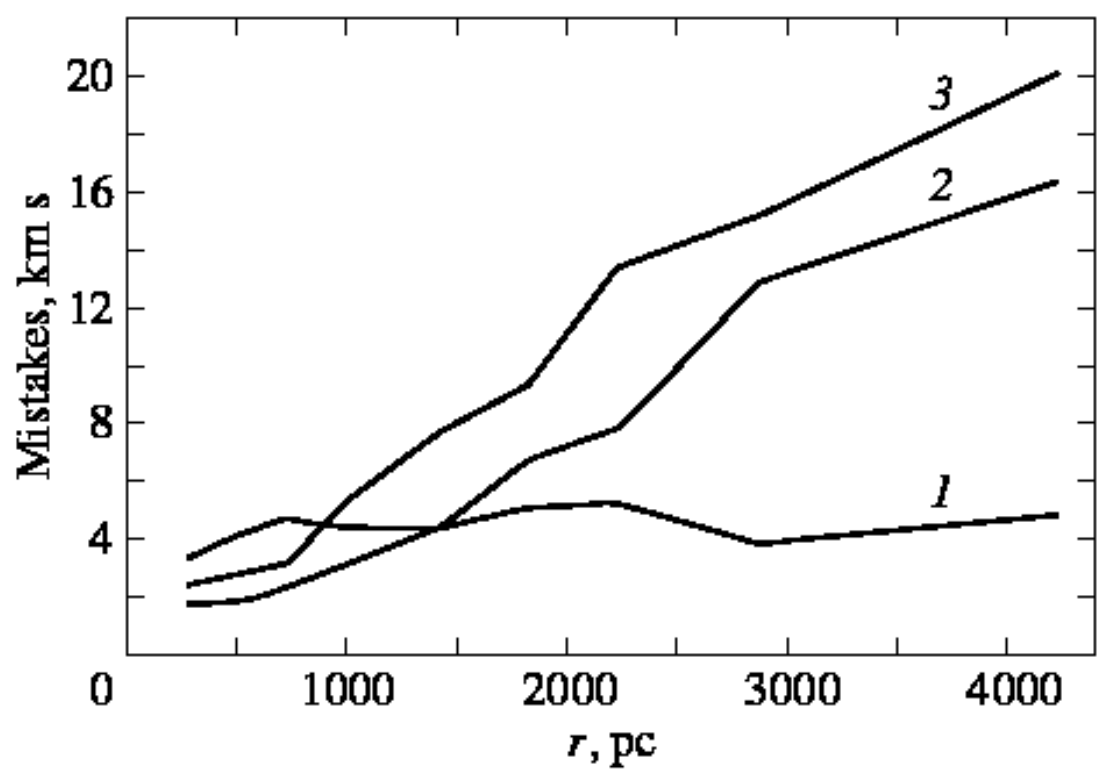

Fig. 2. Mean errors $\sigma_{V_{r}}(1), \sigma_{V_{l}}(3)$, and $\sigma_{V_{b}}(2)$ versus heliocentric distance.

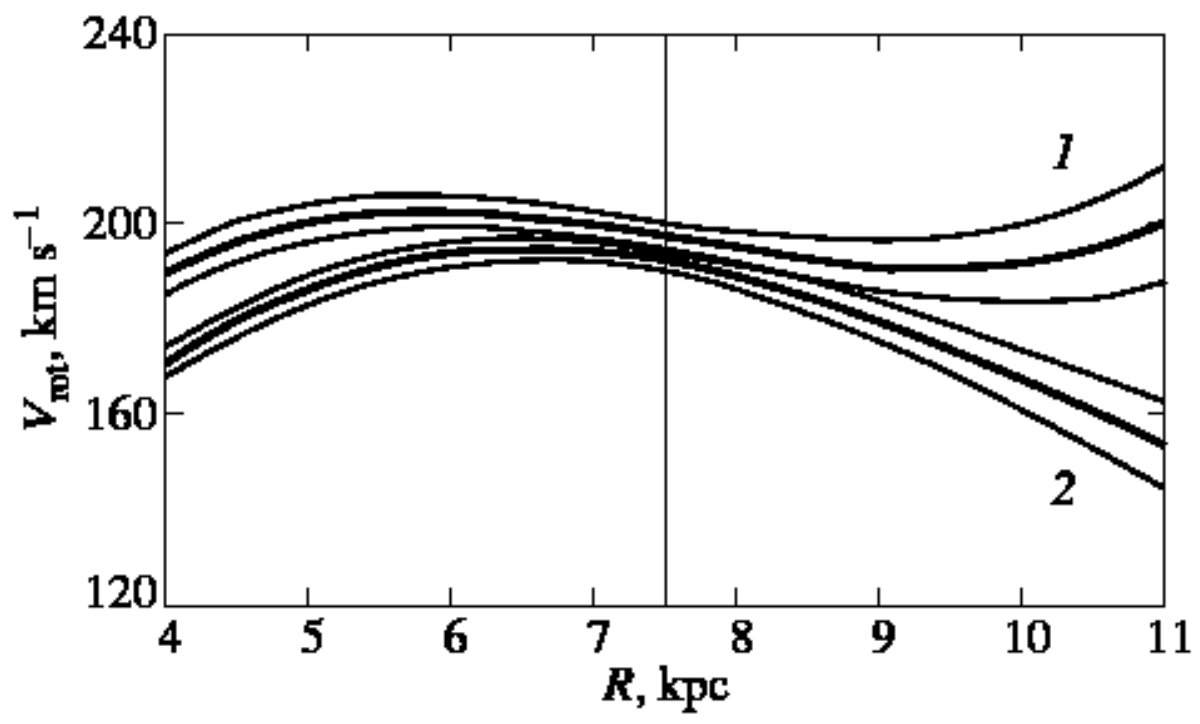

Fig. 3. Galactic rotation curve from clusters within $2.5 \mathrm{kpc}(1)$ and $5 \mathrm{kpc}(2)$ of the Sun. The thin lines mark the boundaries of the $1 \sigma$ confidence intervals; the vertical line indicates the position of $R_{0}=7.4 \mathrm{kpc}$. 

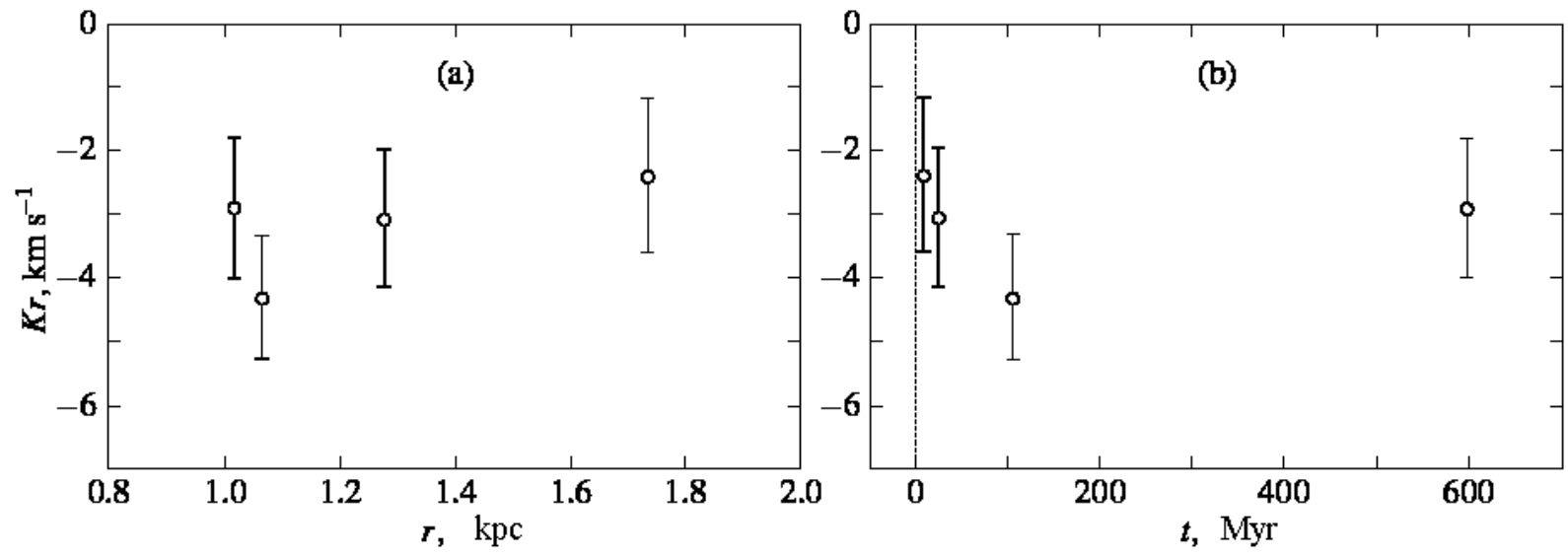

Fig. 4. Linear contraction velocity versus (a) heliocentric distance and (b) mean sample age. 